\title{
S-H OWA Operators with Moment Measure
}

\author{
Jian Wang, ${ }^{1}$ José M. Merigó, ${ }^{2}$ LeSheng Jin ${ }^{1, *}$ \\ ${ }^{1}$ Business School, Nanjing Normal University, Nanjing, People's Republic of \\ China \\ ${ }^{2}$ Department of Management Control and Information Systems, University of \\ Chile, 8330015 Santiago, Chile
}

\begin{abstract}
Step-like or Hurwicz-like ordered weighted averaging (OWA) (S-H OWA) operators connect two fundamental OWA operators, step OWA operators and Hurwicz OWA operators. S-H OWA operators also generalize them and some other well-know OWA operators such as median and centered OWA operators. Generally, there are two types of determination methods for S-H OWA operators: One is from the motivation of some existed mathematical results; the other is by a set of "nonstrict" definitions and often via some intermediate elements. For the second type, in this study we define two sets of strict definitions for Hurwitz/step degree, which are more effective and necessary for theoretical studies and practical usages. Both sets of definitions are useful in different situations. In addition, they are based on the same concept moment of OWA operators proposed in this study, and therefore they become identical in limit forms. However, the Hurwicz/step degree (HD/SD) puts more concerns on its numerical measure and physical meaning, whereas the relative Hurwicz/step degree (rHD/rSD), still being accurate numerically, sometimes is more reasonable intuitively and has larger potential in further studies and practical applications. (c) 2016 Wiley Periodicals, Inc.
\end{abstract}

\section{INTRODUCTION}

A special type of aggregation function (or aggregation operator) with preferences involved is important in large varieties of applications such as decision-making problems, ${ }^{1-3}$ computational intelligence, ${ }^{4-9}$ and information processing. ${ }^{10,11}$ There are many theories about this type that can be found in some part of Yager's decision theory involving preferences ${ }^{1,3,7-9,12-26}$ and some aggregation functions studies. ${ }^{27-33}$ For example, the well-known ordered weighted averaging (OWA) operator ${ }^{25}$ has been widely used in a large variety of areas and applications. . $^{3,9,11-28,34-36}$ We have known that OWA operators can effectively model and generalize many cognitive concepts such as maximum, minimum, and average of an input arguments set. Some other concepts such as median, Hurwicz, and median-like operators can also be generalized by centered OWA operators. ${ }^{7}$ Centered (and centered-like) OWA operators as another branch of OWA operators that are gradually studied and applied in practice. $^{7,12}$

*Author to whom all correspondence should be addressed; e-mail: jls1980@163.com

INTERNATIONAL JOURNAL OF INTELLIGENT SYSTEMS, VOL. 00, 1-16 (2016)

(C) 2016 Wiley Periodicals, Inc.

View this article online at wileyonlinelibrary.com. • DOI 10.1002/int.21829 
The orness/andness measure of OWA operators, also introduced by Yager, ${ }^{25}$ plays a significant role in the researches of OWA operators, ${ }^{7-9,12-15,17-20,25,26}$ including the centered (and centered-like) OWA operators. Generally, as we know, the orness/andness measure reflects the extent of orlike or andlike of the corresponding aggregating result of an ordered argument set under a given OWA operator. Note that the concept orness can also be used to help in determining some centeredlike or Hurwicz-like OWA operators with given preferred parameters. ${ }^{14}$ Step OWA operators $^{26}$ and generalized step OWA operators ${ }^{12}$ play a crucial role in those determination methods for generating step-like and Hurwicz-like OWA operators. ${ }^{14}$ In this sense, step-like and Hurwicz-like OWA operators can be seen as two contrary extreme cases, just like the max and min as extreme cases with respect to their orness degree 1 and 0.

Generally speaking, we can present two classes of determination methods for these "step-like/Hurwitz-like" (S-H) OWA operators. One is to directly derive these OWA operators by some special well-known definitions or backgrounds. For example, the Gaussian-type OWA operators ${ }^{24}$ are motivated by Gaussian distribution, Olympic type of OWA operators ${ }^{26}$ have their own special meaning, and the binomial OWA operator, ${ }^{10}$ still being centered-like, has already successfully shown its application in pattern recognition. ${ }^{10}$ The other is based on some relatively accurate measurements ${ }^{12,14}$ (sometimes via certain "intermediate" elements, which help to determine those OWA operators). This type of determination methods has its own advantages because it can consider two parameters "centered degree and orness degree" provided by different decision makers. That is, we first define a type of step/Hurwitz degree for any OWA operators with any orness/andness degree; then decision makers can decide their wanted step/Hurwitz degree with their preferred orness/andness degree.

In Ref. 14, we defined a type of centered/Hurwitz degree, based on which we can determine infinitely more OWA operators with different orness/andness degree. Its definition is closely connected with the "intermediate" elements—still a group of OWA operators. Though it is sufficiently effective for practical applications, we shall still give a more strict and effective definition of step/Hurwitz degree.

The more important is that, at present we do not have a set of effective and accurate definition/measurement on which the generating methods for S-H OWA can be based. In this study, motivated by the concept moment in physics, we introduce the concept moment for OWA operators with its own meaning and explanations. Actually, this meaning is itself evident, because natural intuition can tell us that the larger moment, the larger Hurwicz degree for an OWA operator, and vice versa.

We shall present two sets of definitions about Hurwicz/step degree (later also called HD/SD). The first set puts more concerns on its numerical measure; that is, it is more near the original physical meaning. However, the second set of definitions, defining the relative Hurwicz/Step degree (later also called $\mathrm{rHD} / \mathrm{rSD}$ ), is more reasonable intuitively and has larger potential in further studies and applications. One of the features of these definitions for $\mathrm{rHD} / \mathrm{rSD}$ is that it can effectively avoid some dilemma or unpleasant situations HD/SD may face, as we shall discuss later. 
The remainder of this study is organized as follows: Section 2 reviews some concepts related to OWA operators. Section 3 briefly reviews four types of generation methods for centered-like or step-like OWA operators and points out their own backgrounds, advantages, and shortcomings, respectively. In Section 4, we propose the concept moment of OWA operators and present a set of definitions for HD/SD. In Section 5, a set of definitions for $\mathrm{rHD} / \mathrm{rSD}$ is proposed and compared with its counterpart in Section 4. Section 6 summarizes the main results and conclusions.

\section{PRELIMINARY}

The well-known OWA operator and orness/andness degree were proposed by Yager $^{25}$ as follows.

DEFINITION 1. ${ }^{25}$ An OWA operator of dimension $n$ is a mapping $F: R^{n} \rightarrow R$ that has an associated weighting vector $\mathbf{w}=\left(w_{1}, w_{2}, \ldots, w_{n}\right)$ having the properties

$$
w_{1}+w_{2}+\cdots+w_{n}=1 ; \quad 0 \leq w_{j} \leq 1 ; \quad j=1,2, \ldots, n
$$

and such that

$$
F_{\mathbf{w}}\left(a_{1}, a_{2}, \ldots, a_{n}\right)=\sum_{j=1}^{n} w_{j} b_{j}
$$

with $b_{j}$ being the jth largest of $a_{i}$.

Using vector form, (1) can be rewritten as

$$
F_{\mathbf{w}}(\mathbf{a})=\mathbf{w b}^{T}
$$

where $\mathbf{a}=\left(a_{1}, a_{2}, \ldots, a_{n}\right)$ is the original arguments vector, whereas $\mathbf{b}=$ $\left(b_{1}, b_{2}, \ldots, b_{n}\right)$ is its ordered form with $b_{1} \geq b_{2} \geq \cdots \geq b_{n}$, and $\mathbf{w}=$ $\left(w_{1}, w_{2}, \ldots, w_{n}\right)$ is the OWA operator.

DEFINITION 2. ${ }^{25}$ The degree of "orness" associated with this operator is defined as

$$
\operatorname{orness}(\mathbf{w})=\frac{1}{n-1} \sum_{i=1}^{n}(n-i) w_{i}=\sum_{i=1}^{n} \frac{n-i}{n-1} w_{i}
$$

The measure of "andness" associated with an OWA operator is the complement of its "orness", and is defined as

$$
\operatorname{andness}(\mathbf{w})=1-\operatorname{orness}(\mathbf{w})
$$

or

$$
\operatorname{andness}(\mathbf{w})=\frac{1}{n-1} \sum_{i=1}^{n}(i-1) w_{i}=\sum_{i=1}^{n} \frac{i-1}{n-1} w_{i} .
$$


PROPOSITION 1. ${ }^{25}$ If the max, min, and average operator correspond to $\mathbf{w}^{*}, \mathbf{w}_{*}$, and $\mathbf{w}_{A}$, respectively, where $\mathbf{w}^{*}=(1,0, \ldots, 0), \mathbf{w}_{*}=(0,0, \ldots, 1)$, and $\mathbf{w}_{A}=$ $(1 / n, 1 / n, \ldots, 1 / n)$, then $\operatorname{orness}\left(\mathbf{w}^{*}\right)=1$, orness $\left(\mathbf{w}_{*}\right)=0$ and $\operatorname{orness}\left(\mathbf{w}_{A}\right)=1 / 2$.

In decision-making problems, max, min, and average operator correspond to fully optimistic decision, fully pessimistic decision, and Laplace decision criterion, respectively. Apart from these, the Hurwicz decision model, $\mathbf{w}_{H(\alpha)}=$ $(\alpha, 0, \ldots, 0,1-\alpha)$, also serves as a class of fundamental OWA operator, where $F_{\mathbf{w}_{H(\alpha)}}\left(a_{1}, a_{2}, \ldots, a_{n}\right)=\alpha \max \left(a_{i}\right)+(1-\alpha) \min \left(a_{i}\right)$ and orness $\left(\mathbf{w}_{H(\alpha)}\right)=\alpha$.

The next proposition shows one particular reverse property related to the orness degree of two OWA operators that are reverse of each other.

PROPOSITION 2. ${ }^{25}$ (Reverse Property). For any OWA weighting vector $\mathbf{w}=\left(w_{1}, w_{2}, \ldots, w_{n}\right)$, orness $(\mathbf{w})=\alpha$, then for the reverse of $\mathbf{w}: \mathbf{w}^{\prime}=$ $\left(w_{1}^{\prime}, w_{2}^{\prime}, \ldots, w_{n}^{\prime}\right)=\left(w_{n}, w_{n-1}, \ldots, w_{1}\right)$,orness $\left(\mathbf{w}^{\prime}\right)=1-\alpha$.

DEFINITION 3. ${ }^{20}$ For an OWA operator $\mathbf{w}=\left(w_{1}, w_{2}, \ldots, w_{n}\right)$, if $\forall i, j, i<j$, implies $w_{i} \leq w_{j}$, then we call $\boldsymbol{w}$ a monotonic increasing OWA operator; reversely, if $\forall i, j, i<j$, implies $w_{i} \geq w_{j}$, then we call $\boldsymbol{w}$ a monotonic decreasing OWA operator. Both of the monotonic increasing and decreasing OWA operators can be collectively referred to as the monotonic OWA operator.

DEFINITION $4 .^{26}$ A step OWA operator of dimension $n \mathbf{w}_{\text {step }}=\left(w_{1}, w_{2}, \ldots, w_{n}\right)$ is of the following form:

$$
\begin{aligned}
& w_{t}=1, \\
& w_{i}=0, \quad i \neq t .
\end{aligned}
$$

With the step OWA operator, we have just one nonzero weight and that is the $k$ th weight, e.g., $\mathbf{w}_{\text {step }}=(0, \cdots, 1, \cdots, 0)$. It is easily seen that the aggregation under this step OWA $F_{\mathbf{w}_{\text {step }}}\left(a_{1}, \cdots, a_{n}\right)=b_{k}$, where $b_{k}$ is the $k$ th largest of the arguments.

Note that $n$-dimensional step OWA operators can only have $n$ different andness degrees. So we could generalize the step OWA operator with this form (depending on its orness/andness): ${ }^{12}$

$$
\mathbf{w}_{\text {step }}=[0,0, \cdots,(1-k), k, \cdots, 0,0] .
$$

DEFINITION 5. ${ }^{12}$ A generalized step OWA operator of dimension $n \mathbf{w}_{\text {step }}=$ $\left(w_{1}, w_{2}, \ldots, w_{n}\right)$ is of the following form:

$$
\begin{aligned}
& w_{t}=1-k, w_{t+1}=k,(k \in[0,1]) \\
& w_{i}=0, i \neq t \text { and } i \neq t+1
\end{aligned}
$$

This type of generalized step OWA operators can take any value in $[0,1]$ as the predefined orness/andness degree. The next theorem presents the method for generating this generalized Step OWA with a given andness degree. 
THEOREM $1 .{ }^{12}$ To generate the $n$-dimensional generalized step OWA $\mathbf{w}_{\text {step }}^{(n)}$ with predefined andness $\alpha$, let

$$
k=(n-1) \alpha-\lfloor(n-1) \alpha\rfloor \text { and } 1-k=\lfloor(n-1) \alpha\rfloor+1-(n-1) \alpha
$$

where $\amalg$ is the greatest integer function, and let

$$
\begin{aligned}
\mathbf{w}_{\text {step }}^{(n)} & =\left(0,0, \ldots, w_{\lfloor(n-1) \alpha+1\rfloor}, w_{\lfloor(n-1) \alpha+2\rfloor}, \ldots, 0,0\right) \\
& =(0,0, \ldots,(1-k), k, \ldots, 0,0),
\end{aligned}
$$

then $\operatorname{andness}\left(\mathbf{w}_{\text {step }}^{(n)}\right)=\alpha$.

Example 1. Generate the generalized step OWA operator $\mathbf{w}_{\text {step }}^{(7)}$ with predefined andness $\alpha=0.8$.

Solution. $\quad k=(n-1) \alpha-\lfloor(n-1) \alpha\rfloor=6 \cdot 0.8-\lfloor 6 \cdot 0.8\rfloor=4.8-4=0.8, \quad 1-$ $k=0.2$

So $w_{\lfloor(n-1) \alpha+1\rfloor}=w_{5}=1-k=0.2, w_{6}=0.8$, thus $\mathbf{w}_{\text {step }}^{(7)}=(0,0,0,0,0.2,0.8,0)$.

\section{THE S-H OWA OPERATORS DERIVED BY SOME KNOWN MATHEMATICAL RESULTS}

In this section, we review some step-like or Hurwicz-like OWA operators that are motivated by or derived from some known results and have many applications (like in image reduction) and good theoretical values.

\subsection{Centered OWA Operators}

Before the introduction of centered OWA operators proposed by Yager, ${ }^{7}$ OWA operators mainly focused on the extent to which the preference is given to larger or smaller arguments. In other words, they seldom consider those general situations where most of weights are put near one particular argument (or position). For example, when orness is 0.5 , this situation reflects a neutral attitudinal character (or decision preference). But we must find that having orness $=0.5$ does not necessarily guarantee preference being given to the arguments which lie in the central position. More generally, suppose orness is $\alpha$; it also does not guarantee that the preference can be placed to the arguments which lie near the $100 \alpha \%$ position (from smaller ones) of that ordered arguments set. Assume the orness is predetermined, we do not know whether most of weights are placed on both "ends" of an ordered arguments vector (like the Hurwicz decision model), or on the "middle or $100 \alpha \%$ position" of that ordered arguments set (like step OWA operators).

DEFINITION 6. ${ }^{7}$ An OWA operator of dimension $n$ is said to be a centered OWA operator if its associated weighting vector $\boldsymbol{w}$ satisfies the following conditions: 
1. Symmetric: $w_{n+1-j}=w_{j}$.

2. Strongly decaying: If $i<j \leq(n+1) / 2$, then $w_{i}<w_{j}$ and if $i>j \geq(n+1) / 2$, then $w_{i}<w_{j}$.

3. Inclusive: $w_{j}>0$.

A weaker edition of condition 2 is defined as follows: ${ }^{7}$

2. Softly decaying: If $i<j \leq(n+1) / 2$, then $w_{i} \leq w_{j}$ and if $i>j \geq(n+1)$ $/ 2$, then $w_{i} \leq w_{j}$.

It is clear that the Centered OWA operators have only one orness degree with 0.5 though it can have different Centered degrees. However, this pioneering definition is very successful and enlightening, because for the first time it systematically and numerically proposes the problem and provides useful way to solve this type of problems.

\subsection{Binomial OWA Operators} follows:

Derived from binomial polynomials, the binomial OWA operator is defined as

DEFINITION $7 .^{10}$ The OWA operator $\mathbf{w}=\left(w_{1}, w_{2}, \ldots, w_{n}\right)$ with the weights such that

$$
w_{i}=\left(\begin{array}{c}
n-1 \\
i-1
\end{array}\right)(1-\alpha)^{i-1} \alpha^{n-i}
$$

is called the binomial OWA operator. Note that it has the constant orness degree: orness $(\mathbf{w})=\alpha$, irrespective of its dimension.

The binomial OWA operator can have different orness degree with parameter $\alpha$ changing and has shown significant application in pattern recognition. ${ }^{10}$ Nevertheless, it lacks the variation in the step-like or Hurwicz-like degrees. ${ }^{14}$

\subsection{Stancu OWA Operators}

As the generalization form of binomial OWA operators and even Hurwicz OWA operators, Stancu OWA operators ${ }^{36}$ can have more variation in the possible Hurwicz-like extent, though with much more complex mathematical structures.

The Stancu OWA operators ${ }^{36}$ are defined as follows: For an OWA operator of dimension $i, \mathbf{u}^{(i)}=\left(u_{i 1}, u_{i 2}, \ldots, u_{i i}\right)(i=1,2, \ldots)$, if its weights satisfy the condition (an empty product denotes 1 )

$$
u_{i j}=\frac{\left(\begin{array}{l}
i-1 \\
i-j
\end{array}\right) \prod_{s=0}^{i-j-1}(t+s \alpha) \prod_{s=0}^{j-2}(1-t+s \alpha)}{\prod_{s=0}^{i-2}(1+s \alpha)}
$$

with $t \in[0,1], \alpha \geq 0$ being two parameters, then it is called a Stancu OWA operator. 
It is important that the orness of Stancu OWA operators is just the parameter $t$ irrespective of $\alpha(\alpha \geq 0)$ and dimension $i(i=1,2, \ldots)$. It is also very significant that average OWA operators, binomial OWA operators, and Hurwicz OWA operators are special cases of Stancu OWA operators with parameters $(t=0,5 ; \alpha=0.5)$, $(t=$ orness $; \alpha=0)$ and $(t=$ orness $; \alpha=+\infty)$, respectively. ${ }^{36}$

We can find that sometimes it is not easy to control and get the desired Hurwiczlike degrees due to the complexity of Stancu polynomials. Apart from this, it cannot effectively generalize step-like OWA operators.

\subsection{Piled OWA Operators}

The concept "piled OWA operator" 14 actually is a set of definitions and OWA determination mechanisms. The piled OWA operators can effectively generalize the centered OWA operators and also connect the generalized step OWA operators with the Hurwicz OWA operators with the given orness degree. They can also provide controllable algorithms to generate infinitely more step-like or Hurwicz-like OWA operators with two preferred parameters provided by decision makers: (1) orness degree and (2) step-like or Hurwicz-like degree.

It is clear that this type of piled OWA operators has more flexibility and is more controllable under the preferences of different decision makers. However, there still does not exist a strict measurement for different definitions to be based on.

Remark. The structures and properties of some dynamic families of OWA operators like Stancu OWA operators are quite complex mathematically. However, although those dynamic families are very interesting and have less or more sense of "centered" style, it is not easy of them to be used in all of related applications. More seriously, at present there are no strict definitions to measure the degree of "centering." But motivated from some physics concepts, we proposed the moment measure, step/Hurwicz degree, relative step/Hurwicz Degree and gave some mathematical properties of them, which can present a strict and standard definition for "centering" as we will see in the next section.

\section{THE MOMENT, STEP DEGREE, AND HURWICZ DEGREE OF OWA OPERATORS}

As we discussed before, there needs a strict measurement on which different step degree definitions can be based. The concept moment (M) is naturally a reasonable and effective measurement for further possible definitions. Thus, before defining step degree (SD), and Hurwicz degree (HD) of OWA operators, we shall present some necessary definitions about the moment $(\mathrm{M})$ of OWA operators first.

Definition 8 (Moment). For any OWA operators of dimension $n, \quad \mathbf{w}=$ $\left(w_{1}, w_{2}, \ldots, w_{n}\right)$ with andness $\alpha$, its moment $(M)$ is defined as follows:

$$
M(\mathbf{w})=\sum_{i=1}^{n}\left(w_{i} \cdot\left|\frac{i-1}{n-1}-\alpha\right|\right)
$$


Remark. An equivalent definition using "orness" can be written as for any OWA operators of dimension $n, \mathbf{w}=\left(w_{1}, w_{2}, \ldots, w_{n}\right)$ with orness $\alpha$. Its moment is defined as follows:

$$
M^{\prime}(\mathbf{w})=\sum_{i=1}^{n}\left(w_{i} \cdot\left|\frac{n-i}{n-1}-\alpha\right|\right)
$$

DEFINITION 9. For any OWA operators of dimension $n, \mathbf{w}=\left(w_{1}, w_{2}, \ldots, w_{n}\right)$ with andness $\alpha$, its left moment (LM) is defined as follows:

$$
\mathrm{LM}(\mathbf{w})=\sum_{i=1}^{t}\left(w_{i} \cdot\left|\frac{i-1}{n-1}-\alpha\right|\right)
$$

such that $\frac{t-1}{n-1} \leq \alpha$ and $\frac{t}{n-1} \geq \alpha$.

DEFINITION 10. For any OWA operators of dimension $n, \mathbf{w}=\left(w_{1}, w_{2}, \ldots, w_{n}\right)$ with andness $\alpha$, its right moment (RM) is defined as follows:

$$
\operatorname{RM}(\mathbf{w})=\sum_{i=t+1}^{n}\left(w_{i} \cdot\left|\frac{i-1}{n-1}-\alpha\right|\right)
$$

such that $\frac{t-1}{n-1} \leq \alpha$ and $\frac{t}{n-1} \geq \alpha$.

Proposition 3. For any OWA operators of dimension $n, \mathbf{w}=\left(w_{1}, w_{2}, \ldots, w_{n}\right)$ with andness $\alpha$, we have $M(\mathbf{w})=\mathrm{LM}(\mathbf{w})+\mathrm{RM}(\mathbf{w})$.

Example 2. Given the OWA operator $\mathbf{w}=(0.15,0.3,0.15 .0 .4)$ with andness 0.6 , it has $\mathrm{M}, \mathrm{LM}$, and RM as follows:

$$
\begin{aligned}
M(\mathbf{w})= & \sum_{i=1}^{4}\left(w_{i} \cdot\left|\frac{i-1}{n-1}-0.6\right|\right) \\
= & (0.15) \cdot 0.6+(0.3) \cdot\left(0.6-\frac{1}{3}\right)+(0.15) \cdot\left(\frac{2}{3}-0.6\right) \\
& +(0.4) \cdot(1-0.6) \\
= & 0.09+0.08+0.01+0.16=0.34 .
\end{aligned}
$$

Since $\frac{1}{3} \leq 0.6$ and $\frac{2}{3} \geq 0.6$, then $t=2$; thus

$$
\begin{aligned}
\operatorname{LM}(\mathbf{w}) & =\sum_{i=1}^{2}\left(w_{i} \cdot\left|\frac{i-1}{n-1}-0.6\right|\right) \\
& =(0.15) \cdot 0.6+(0.3) \cdot\left(0.6-\frac{1}{3}\right)=0.09+0.08=0.17
\end{aligned}
$$

International Journal of Intelligent Systems $\quad$ DOI 10.1002/int 


$$
\begin{aligned}
\operatorname{RM}(\mathbf{w}) & =\sum_{i=2+1}^{4}\left(w_{i} \cdot\left|\frac{i-1}{n-1}-0.6\right|\right) \\
& =(0.15) \cdot\left(\frac{2}{3}-0.6\right)+(0.4) \cdot(1-0.6)=0.01+0.16=0.17
\end{aligned}
$$

From Example 1, we can find the following fact:

THEOREM 2. For any OWA operators of dimension $n, \mathbf{w}=\left(w_{1}, w_{2}, \ldots, w_{n}\right)$ with andness $\alpha$, we have $\mathrm{LM}(\mathbf{w})=\mathrm{RM}(\mathbf{w})=\frac{1}{2} M(\mathbf{w})$.

Proof. Since andness $(\mathbf{w})=\alpha=\sum_{i=1}^{n} \frac{i-1}{n-1} w_{i}$, then when $t$ satisfies $\frac{t-1}{n-1} \leq \alpha$ and $\frac{t}{n-1} \geq \alpha$

$$
\begin{aligned}
\mathrm{RM}(\mathbf{w})-\mathrm{LM}(\mathbf{w}) & =\sum_{i=t+1}^{n}\left(w_{i} \cdot\left|\frac{i-1}{n-1}-\alpha\right|\right)-\sum_{i=1}^{t}\left(w_{i} \cdot\left|\frac{i-1}{n-1}-\alpha\right|\right) \\
& =\sum_{i=t+1}^{n}\left(w_{i} \cdot\left(\frac{i-1}{n-1}-\alpha\right)\right)+\sum_{i=1}^{t}\left(w_{i} \cdot\left(\frac{i-1}{n-1}-\alpha\right)\right) \\
& =\sum_{i=1}^{n} w_{i} \cdot\left(\frac{i-1}{n-1}-\alpha\right)=\left(\sum_{i=1}^{n} \frac{i-1}{n-1} w_{i}\right)-\alpha=0
\end{aligned}
$$

It is clear that $M(\mathbf{w}) \geq 0$; and $M(\mathbf{w})=0$ if and only if $\mathbf{w}$ is a step OWA operator (specially note that here it is not "generalized step OWA operator"; recall Definitions 4 and 5). For any OWA operator $\mathbf{w}$ of dimension $n$, generally we cannot always have $\min (M(\mathbf{w}))=0$; but when $n$ is large, the minimum moment it can take is very near zero, as later we shall see.

However, for the maximum moment it can take, we have the following important property.

THEOREM 3. For any OWA operator of dimension $n, \mathbf{w}=\left(w_{1}, w_{2}, \ldots, w_{n}\right)$ with andness $\alpha$, its moment has a maximum value: $\max (M(\mathbf{w}))=M\left(\mathbf{w}_{H(1-\alpha)}\right)=2 \alpha(1-$ $\alpha)$ where $\mathbf{w}_{H(1-\alpha)}=(1-\alpha, 0, \ldots, 0, \alpha)$, the Hurwicz OWA operator with andness $\alpha$ (orness $1-\alpha)$.

Proof. First, by Definition 8, it is clear that $M\left(\mathbf{w}_{H(1-\alpha)}\right)=2 \alpha(1-\alpha)$ and then

$$
\mathrm{LM}\left(\mathbf{w}_{H(1-\alpha)}\right)=\operatorname{RM}\left(\mathbf{w}_{H(1-\alpha)}\right)=\alpha(1-\alpha)
$$

Second, suppose there is an OWA operator $\mathbf{v}=\left(v_{1}, v_{2}, \ldots, v_{n}\right)$ also with andness $\alpha$, and its moment is larger than $M\left(\mathbf{w}_{H(1-\alpha)}\right)$, i.e., $M(\mathbf{v})>M\left(\mathbf{w}_{H(1-\alpha)}\right)$. This also implies that

$$
\mathrm{LM}(\mathbf{v})>\operatorname{LM}\left(\mathbf{w}_{H(1-\alpha)}\right) \quad \text { and } \quad \mathrm{RM}(\mathbf{v})>\operatorname{RM}\left(\mathbf{w}_{H(1-\alpha)}\right) .
$$


When $t$ satisfies $\frac{t-1}{n-1} \leq \alpha$ and $\frac{t}{n-1} \geq \alpha$, if (8) holds, then we can easily see that $\sum_{i=1}^{t} v_{i}>1-\alpha$ and $\sum_{i=t+1}^{n} v_{i}>\alpha$, which mean $1=\sum_{i=1}^{n} v_{i}=\sum_{i=1}^{t} v_{i}+\sum_{i=t+1}^{n} v_{i}>$ $1-\alpha+\alpha=1$.

It is contradictory and then we must have $\max (M(\mathbf{w}))=M\left(\mathbf{w}_{H(1-\alpha)}\right)=$ $2 \alpha(1-\alpha)$.

Proposition 4. For any OWA operator of dimension $n, \mathbf{w}=\left(w_{1}, w_{2}, \ldots, w_{n}\right)$ with any andness degree, it takes the (global) maximum moment 0.5 when andness $\alpha=0.5$.

Remark. Though it is the "global" maximum, it is only one of those maximum moments; that is, it has no significant meaning in practice because our concern is only on the extent to which more weights are put near the $100 \% \alpha$ position (from smaller ones).

Proposition 5. For two OWA operators of dimension $n, \mathbf{u}=\left(u_{1}, u_{2}, \ldots, u_{n}\right)$ and $\mathbf{v}=\left(v_{1}, v_{2}, \ldots, v_{n}\right)$, with $M(\mathbf{u})=a$ and $M(\mathbf{v})=b$, if $\mathbf{w}=\lambda \mathbf{u}+(1-\lambda) \mathbf{v}$, then $M(\mathbf{w})=\lambda a+(1-\lambda) b$.

The definitions and related properties above presented are nonnormalized and hence are not very suitable for practical applications. It is natural that we must provide the subsequent standard definitions.

DEFINITION 11 (Hurwicz Degree). For any OWA operator of dimension $n(n>2)$, $\mathbf{w}=\left(w_{1}, w_{2}, \ldots, w_{n}\right)$ with andness $\alpha(\alpha \in(0,1))$, its $H D$ is defined as

$$
\mathrm{HD}(\mathbf{w})=\frac{M(\mathbf{w})}{M\left(\mathbf{w}_{H(1-\alpha)}\right)}=\frac{M(\mathbf{w})}{2 \alpha(1-\alpha)}
$$

DEFINITION 12 (Step Degree). For any OWA operator of dimension $n(n>2)$, $\mathbf{w}=\left(w_{1}, w_{2}, \ldots, w_{n}\right)$ with andness $\alpha(\alpha \in(0,1))$, its $S D$ is defined as

$$
\mathrm{SD}(\mathbf{w})=1-\mathrm{HD}(\mathbf{w})=1-\frac{M(\mathbf{w})}{M\left(\mathbf{w}_{H(1-\alpha)}\right)}=1-\frac{M(\mathbf{w})}{2 \alpha(1-\alpha)} .
$$

Remarks.

1. From Definitions 11 and 12, we normalized the moment for practical measurements. For any OWA operator of dimension $n(n>2), \mathbf{w}=\left(w_{1}, w_{2}, \ldots, w_{n}\right), \operatorname{HD}(\mathbf{w}) \in[0,1]$, $\mathrm{SD}(\mathbf{w}) \in[0,1]$, and $\mathrm{HD}(\mathbf{w})+\mathrm{SD}(\mathbf{w})=1$. One can see it is reasonable and effective in practice both intuitively and numerically.

2. We need not consider the cases where andness is 0 or 1 since they are trivial, i.e., they have only one form.

Proposition 6. Any step OWA operator $\mathbf{w}_{\text {step }}$ has $\mathrm{SD}\left(\mathbf{w}_{\text {step }}\right)=1$, whereas any Hurwicz OWA operator $\mathbf{w}_{H(\alpha)}$ has $\operatorname{HD}\left(\mathbf{w}_{H(\alpha)}\right)=1$. 
Proposition 7. For two OWA operators of dimension $n$ with the same andness, $\mathbf{u}=$ $\left(u_{1}, u_{2}, \ldots, u_{n}\right)$ and $\mathbf{v}=\left(v_{1}, v_{2}, \ldots, v_{n}\right)$, with $\mathrm{HD}(\mathbf{u})=k$ and $\mathrm{HD}(\mathbf{v})=l$ fulfills, if $\mathbf{w}=\lambda \mathbf{u}+(1-\lambda) \mathbf{v}$, then $\operatorname{HD}(\mathbf{w})=\lambda k+(1-\lambda) l$.

Example 3. Given the OWA operator $\mathbf{w}=(0.15,0.3,0.15,0.4)$ with andness $\alpha=$ 0.6 , it has $M(\mathbf{w})=0.34$ and $M\left(\mathbf{w}_{H(1-\alpha)}\right)=2 \alpha(1-\alpha)=0.48$, and

$$
\mathrm{HD}(\mathbf{w})=\frac{M(\mathbf{w})}{M\left(\mathbf{w}_{H(1-\alpha)}\right)}=\frac{0.34}{0.48}=\frac{17}{24}, \quad \mathrm{SD}(\mathbf{w})=1-\mathrm{HD}(\mathbf{w})=\frac{7}{24}
$$

For an OWA operator w, larger SD means more weights are put near the $100 \% \alpha$ position from $w_{n}$ to $w_{1}$; larger HD means more weighs are proportionally (according to $\alpha$ ) split and put near two end $w_{1}$ and $w_{n}$. Thus, with this flexible and controllable mechanism, we can device many accurate algorithms to generate infinite more S-H OWA operators with given preferences of decision makers.

\section{THE RELATIVE STEP DEGREE AND RELATIVE HURWICZ DEGREE OF OWA OPERATORS}

From Theorem 2 and some symmetrical properties, we can present some algorithms for generating S-H OWA operators with given orness/andness and SD/HD. The idea within the method for obtaining piled OWA operators ${ }^{14}$ can also be used as one that we shall improve or modify in later works. Next, we shall present a set of new definitions that are more accurate and reasonable in some sense. We first see a simple example with some possible dilemmas or unpleasant situations.

For a four-dimensional OWA operators with andness $\alpha=0.5$, if it is Hurwicz OWA, $\mathbf{w}_{H(1-\alpha)}$, it must have $\operatorname{HD}\left(\mathbf{w}_{H(1-\alpha)}\right)=1$. But if it is a generalized step OWA (also as median in this case), $\mathbf{w}_{\text {step }}=(0,0.5,0.5,0)$, we cannot have $\operatorname{HD}\left(\mathbf{w}_{\text {step }}\right)=0$ under Definition 11. We only have

$$
\mathrm{HD}\left(\mathbf{w}_{\text {step }}\right)=\frac{M(\mathbf{w})}{M\left(\mathbf{w}_{H(1-\alpha)}\right)}=\frac{M(\mathbf{w})}{2 \alpha(1-\alpha)}=\frac{0.5 \cdot(1 / 6) \cdot 2}{0.5}=\frac{1}{3}
$$

We shall see that this is the minimum HD (also minimum moment), a fourdimensional OWA with andness 0.5 can take. In some situations, we always wish to get $\mathrm{HD}\left(\mathbf{w}_{\text {step }}\right)=0$ since it is the very "median" as possible as we can make.

DEFINITION 13. ${ }^{14}$ We denote a generalized step OWA operator with orness $\alpha$ as $\mathbf{s}_{\alpha}$. Obviously, maximum operator $\mathbf{w}^{*}=\mathbf{s}_{1}$, minimum operator $\mathbf{w}_{*}=\mathbf{s}_{0}$, and median operator $\mathbf{w}_{m d}=\mathbf{s}_{0.5}$.

According to Definition 13, the Hurwicz OWA operator $\mathbf{w}_{H(1-\alpha)}$ can also be expressed as the convex combination form: $\mathbf{w}_{H(1-\alpha)}=\alpha \mathbf{s}_{0}+(1-\alpha) \mathbf{s}_{1}$, whose $\operatorname{HD}\left(\mathbf{w}_{H(1-\alpha)}\right)=1$. If $\mathbf{s}_{1-\alpha}$ is just a step OWA operator with andness $\alpha$, then $\operatorname{HD}\left(\mathbf{s}_{1-\alpha}\right)=0$; if not, then its HD is not equal to 0 . If $\mathbf{s}_{1-\alpha}$ is not a step OWA operator, i.e., it has the form $\mathbf{s}_{1-\alpha}=\left[0,0, \ldots, w_{\lfloor(n-1) \alpha+1\rfloor}, w_{\lfloor(n-1) \alpha+2\rfloor}, \ldots, 0,0\right]$ 
(see Theorem 1), then it has the minimum HD (or moment) as next we shall prove. We denote this minimum HD ( $\mathbf{s}_{1-\alpha}$ takes) by $m$, and $(m, 1]$ is then the interval these OWA operators (with andness $\alpha$ and same dimension) can take. Though it is clear that when $n$ is very large $m$ will decrease near zero, sometimes we need a set of more suitable definitions, from which we shall get a consistent concept, i.e., any generalized step OWA operator $\mathbf{s}_{1-\alpha}$ (or $\mathbf{s}_{\alpha}$ by symmetry) has zero HD.

THEOREM 4. For any OWA operator of dimension $n, \mathbf{w}=\left(w_{1}, w_{2}, \ldots, w_{n}\right)$ with andness $\alpha$, its moment has a minimum value: $\min (M(\mathbf{w}))=M\left(\mathbf{s}_{1-\alpha}\right)=\frac{2 k(1-k)}{n-1}$ where $k=(n-1) \alpha-\lfloor(n-1) \alpha\rfloor$.

Proof. First, we can see that

$$
M\left(\mathbf{s}_{1-\alpha}\right)=(1-k) \cdot\left|\frac{\lfloor(n-1) \alpha\rfloor}{n-1}-\alpha\right|+k \cdot\left|\frac{\lfloor(n-1) \alpha+1\rfloor}{n-1}-\alpha\right| ;
$$

and by Theorem 2, we also have

$$
\operatorname{LM}\left(\mathbf{s}_{1-\alpha}\right)=(1-k) \cdot\left|\frac{\lfloor(n-1) \alpha\rfloor}{n-1}-\alpha\right|=k \cdot\left|\frac{\lfloor(n-1) \alpha+1\rfloor}{n-1}-\alpha\right|=\operatorname{RM}\left(\mathbf{s}_{1-\alpha}\right)
$$

And note that

$$
(1-k) \cdot\left|\frac{\lfloor(n-1) \alpha\rfloor}{n-1}-\alpha\right|=(1-k) \cdot\left(\alpha-\frac{\lfloor(n-1) \alpha\rfloor}{n-1}\right)=(1-k) \frac{k}{n-1}
$$

(by Theorem 1).

Second, (similar to the proof in Theorem 3), suppose there is an OWA operator $\mathbf{v}=\left(v_{1}, v_{2}, \ldots, v_{n}\right)$ also with andness $\alpha$, and its moment is smaller than $M\left(\mathbf{s}_{1-\alpha}\right)$, i.e., $M(\mathbf{v})<M\left(\mathbf{s}_{1-\alpha}\right)$. This also implies that

$$
\operatorname{LM}(\mathbf{v})<\operatorname{LM}\left(\mathbf{s}_{1-\alpha}\right) \text { and } \operatorname{RM}(\mathbf{v})<\operatorname{RM}\left(\mathbf{s}_{1-\alpha}\right) .
$$

When $t$ satisfies $\frac{t-1}{n-1} \leq \alpha$ and $\frac{t}{n-1} \geq \alpha$, then we can easily see that

$$
\sum_{i=1}^{t} v_{i}<1-k \text { and } \sum_{i=t+1}^{n} v_{i}<k
$$

which means

$$
1=\sum_{i=1}^{n} v_{i}=\sum_{i=1}^{t} v_{i}+\sum_{i=t+1}^{n} v_{i}<1-k+k=1
$$

It is contradictory and then we must have $\min (M(\mathbf{w}))=M\left(\mathbf{s}_{1-\alpha}\right)=\frac{2 k(1-k)}{n-1}$. 
COROLLARY 1. For the generalized step OWA operator of dimension $n$, when $n \rightarrow \infty$, $M\left(\mathbf{s}_{1-\alpha}\right) \rightarrow 0$.

We now present the following alternative definitions that in some sense are more reasonable and have their own special usages and properties.

DeFinition 14 (relative Hurwicz Degree). For any OWA operator of dimension $n$ $(n>2), \mathbf{w}=\left(w_{1}, w_{2}, \ldots, w_{n}\right)$ with andness $\alpha$, its relative Hurwicz degree $(r H D)$ is defined as

$$
\operatorname{rHD}(\mathbf{w})=\frac{M(\mathbf{w})-M\left(\mathbf{s}_{1-\alpha}\right)}{M\left(\mathbf{w}_{H(1-\alpha)}\right)-M\left(\mathbf{s}_{1-\alpha}\right)}
$$

DEFINITION 15 (relative Step Degree). For any OWA operator of dimension $n(n>2)$, $\mathbf{w}=\left(w_{1}, w_{2}, \ldots, w_{n}\right)$ with andness $\alpha$, its relative step degree $(r S D)$ is defined as

$$
\begin{aligned}
\operatorname{rSD}(\mathbf{w}) & =1-\operatorname{rHD}(\mathbf{w}) \\
& =1-\frac{M(\mathbf{w})-M\left(\mathbf{s}_{1-\alpha}\right)}{M\left(\mathbf{w}_{H(1-\alpha)}\right)-M\left(\mathbf{s}_{1-\alpha}\right)} \\
& =\frac{M\left(\mathbf{w}_{H(1-\alpha)}\right)-M(\mathbf{w})}{M\left(\mathbf{w}_{H(1-\alpha)}\right)-M\left(\mathbf{s}_{1-\alpha}\right)}
\end{aligned}
$$

Remark. It is clear that for any dimension and andness degree, $\operatorname{rHD}\left(\mathbf{w}_{H(1-\alpha)}\right)=1$ and $\operatorname{rHD}\left(\mathbf{s}_{1-\alpha}\right)=0$.

Proposition 8. For two OWA operators of dimension $n$ with the same andness, $\mathbf{u}=\left(u_{1}, u_{2}, \ldots, u_{n}\right)$ and $\mathbf{v}=\left(v_{1}, v_{2}, \ldots, v_{n}\right)$, with $\operatorname{rHD}(\mathbf{u})=k$ and $\operatorname{rHD}(\mathbf{v})=l$, if $\mathbf{w}=\lambda \mathbf{u}+(1-\lambda) \mathbf{v}$, then $\operatorname{rHD}(\mathbf{w})=\lambda k+(1-\lambda) l$.

Proposition 9 (Symmetricity). For any OWA operator $\mathbf{w}=\left(w_{1}, w_{2}, \ldots, w_{n}\right)$, and its reverse $\mathbf{w}^{\prime}=\left(w_{1}^{\prime}, w_{2}^{\prime}, \ldots, w_{n}^{\prime}\right)=\left(w_{n}, w_{n-1}, \ldots, w_{1}\right), \quad M(\mathbf{w})=M\left(\mathbf{w}^{\prime}\right)$; $\mathrm{HD}(\mathbf{w})=\mathrm{HD}\left(\mathbf{w}^{\prime}\right) ; \operatorname{rHD}(\mathbf{w})=\operatorname{rHD}\left(\mathbf{w}^{\prime}\right)$.

\section{Example 4.}

1. For the Hurwicz OWA operator $\mathbf{w}_{H(0.5)}$ and generalized step OWA operator $\mathbf{s}_{0.5}$ of dimension 4 with andness 0.5 , respectively,

$$
M\left(\mathbf{w}_{H(0.5)}\right)=2 \cdot 0.5 \cdot(0.5)=0.5 ; \quad M\left(\mathbf{s}_{0.5}\right)=2 \cdot 0.5 \cdot(1 / 6)=1 / 6
$$

2. For the average OWA operator of dimension $4, \mathbf{w}=(0.25,0.25,0.25,0.25)$, we can get

$$
\begin{gathered}
M(\mathbf{w})=2 \cdot(0.25 \cdot(0.5)+0.25 \cdot(1 / 6))=1 / 3 \\
\operatorname{rHD}(\mathbf{w})=\frac{M(\mathbf{w})-M\left(\mathbf{s}_{1-0.5}\right)}{M\left(\mathbf{w}_{H(1-0.5)}\right)-M\left(\mathbf{s}_{1-0.5}\right)}=\frac{1 / 3-1 / 6}{0.5-1 / 6}=0.5=\mathrm{rSD}(\mathbf{w}),
\end{gathered}
$$

while $\operatorname{HD}(\mathbf{w})=2 / 3$; 
3. Given the OWA operator $\mathbf{w}=(0.15,0.3,0.15,0.4)$ with andness $\alpha=0.6$, it has $M(\mathbf{w})=$ 0.34 and we have $M\left(\mathbf{w}_{H(1-\alpha)}\right)=2 \alpha(1-\alpha)=2 \cdot(0.6) \cdot(0.4)=0.48$, and $M\left(\mathbf{s}_{1-0.6}\right)=$ $2 \cdot 0.2 \cdot(4 / 15)=8 / 75=0.107$.

$$
\operatorname{rHD}(\mathbf{w})=\frac{M(\mathbf{w})-M\left(\mathbf{s}_{1-0.6}\right)}{M\left(\mathbf{w}_{H(1-0.6)}\right)-M\left(\mathbf{s}_{1-0.6}\right)}=\frac{0.34-0.107}{0.48-0.107}=0.6247
$$

Recall that in Example 3, $\operatorname{HD}(\mathbf{w})=\frac{M(\mathbf{w})}{M\left(\mathbf{w}_{H(1-\alpha)}\right)}=\frac{0.34}{0.48}=\frac{17}{24}=0.7083$.

Remark. In Examples 3 and 4(3), though we get different HD and rHD, in practice both of them are reasonable in some sense, and then provide more choices for practitioners in different situations. In addition, when dimension $n$ is large, this difference will become small. Figuratively speaking, it seems a narrow "hollow" within $\mathbf{s}_{1-\alpha}$ if it is under the rHD/rSD definition, whereas it is always "full" under the HD/SD definition.

We present two types of the simplest generation methods for the S-H OWA operator with given HD/SD and rHD/rSD.

Suppose we need to obtain an OWA operator of dimension 4 with andness 0.5 and HD 0.5 (or rHD 0.5). By "taking the middle position," if it is based on the $\mathrm{HD} / \mathrm{SD}$ definition, we may get

$$
\begin{aligned}
\mathbf{w} & =0.5\left(\mathbf{s}_{0.25}+\mathbf{s}_{0.75}\right) \\
& =0.5((0.25,0.75,0,0)+(0,0,0.75,0.25)) \\
& =(0.125,0.375,0.375,0.125)
\end{aligned}
$$

For another generation choice, suppose now we are under the rHD/rSD definition; and by "taking the average of the two extreme cases," we can get

$$
\mathbf{w}=0.5 \mathbf{w}_{H(1-0.5)}+0.5 \mathbf{s}_{1-0.5}=(0.25,0.25,0.25,0.25)
$$

Based on these ideas mentioned above, we can easily devise several suitable and more complex methods for generating S-H OWA operators with given preferences.

In later studies, we shall analyze and present more related properties, generation algorithms, adjustment methods, and applications about S-H OWA operators involving $\mathrm{HD} / \mathrm{SD}$ and $\mathrm{rHD} / \mathrm{rSD}$.

\section{CONCLUSIONS}

In this study, we have reviewed and further summarized some general concepts of Hurwicz-, step-, or centered-like OWA operators, and they can connectively called S-H OWA operators. We have listed four types of existed generating and defining methods and then pointed out each importance and possible shortcoming.

By introducing the concept moment of OWA operators, we further have presented two sets of definitions to give standard measurements for determining and 
generating S-H OWA operators. In detail, we have defined HD/SD and rHD/rSD. We have also proposed several of their properties. Both sets of definitions are suitable for different situations. Furthermore, for generating OWA operators with the same HD and rHD, respectively, generally we get two different OWA operators, though with large dimension this difference must gradually become very tiny.

Although both sets of definitions are based on the same concept moment of OWA operators, the HD/SD puts more concerns on its numerical measure, whereas the $\mathrm{rHD} / \mathrm{rSD}$ is more reasonable intuitively and has larger potential in further theoretical studies and applications.

\section{Acknowledgments}

The authors are very grateful to Prof. Jana Špirková for verifying and examining the logical proofs and correcting typos in this paper.

\section{References}

1. Liu XW, Da QL. A decision tree solution considering the decision maker's attitude. Fuzzy Sets Syst 2005;152:437-454.

2. Merigó JM, Gil-Lafuente AM. New decision-making techniques and their application in the selection of financial products. Inform Sci 2010;180(11):2085-2094.

3. Wang YM, Luo Y, Hua ZS. Aggregating preference rankings using OWA operator weights. Inform Sci 2007;177:3356-3363.

4. Dujmovic JJ. Continuous preference logic for system evaluation. IEEE Trans Fuzzy Syst 2007;15(6):1082-1099.

5. Dujmovic JJ. LSP method and its use for evaluation of Java IDEs. Int J Approx Reason 2006 41:3-22.

6. Filev D, Yager RR. On the issue of obtaining OWA operator weights. Fuzzy Sets Syst 1998;94:157-169.

7. Yager RR. Centered OWA operators. Soft Comput 2007;11:631-639.

8. Yager RR, Filev DP. Parameterized "andlike" and "orlike" OWA operators. Int J Gen Syst 1994;22:297-316.

9. Yager RR, Kacprzyk J, Beliakov G. Recent developments on the ordered weighted averaging operators: Theory and practice. Berlin: Springer-Verlag; 2011.

10. Leon T, Zuccarello P, Ayala G, de Ves E, Domingo J. Applying logistic regression to relevance feedback in image retrieval systems. Pattern Recognit 2007;40:2621-2632.

11. Paternain D, Fernandeza J, Bustince H, Mesiar R, Beliakov G. Construction of image reduction operators using averaging aggregation functions. Fuzzy Sets Syst 2015;261:87111.

12. Jin L. Some properties and representation methods for ordered weighted averaging operators. Fuzzy Sets Syst 2015;261:60-86.

13. Jin L, Kalina M, Qian G. Fuzzy orness measure and new orness axioms. Kybernetika 2015;51:712-723.

14. Jin L, Qian G. On obtaining piled OWA operators. Int J Intell Syst 2015;30:520-536.

15. Jin L, Qian G. OWA Generation function and some adjustment methods for OWA operators with application. IEEE Trans Fuzzy Syst.2016;24(1):168-178.

16. Kishor A, Singh AK, Pal NR. Orness measure of OWA operators: a new approach. IEEE Trans Fuzzy Syst.2014;22(4):1039-1045.

17. Liu XW. On the properties of equidifferent OWA operator. Int J Approx Reason 2006;43:90_ 107. 
18. Liu XW. Models to determine parameterized ordered weighted averaging operators using optimization criteria. Inform Sci 2012;190:27-55.

19. Liu XW. An orness measure for quasi-arithmetic means. IEEE Trans Fuzzy Syst 2006;16(6):837-848.

20. Liu XW, Chen LH. On the properties of parametric geometric OWA operator. Int J Approx Reason 2004;35:163-178.

21. Mesiar R, Stupňanová A, Yager RR. Generalizations of OWA operators. IEEE Trans Fuzzy Syst 2015;23:2154-2162.

22. Torra V. The weighted OWA operator. Int J Intell Syst 1997;12:153-166.

23. Xiong W, Liu H. An axiomatic foundation for Yager's decision theory. Int J Intell Syst 2014;29:365-387.

24. Xu ZS. An overview of methods for determining OWA weights. Int J Intell Syst 2005;20(8):843-865.

25. Yager RR. On ordered weighted averaging aggregation operators in multicriteria decision making. IEEE Trans Syst Man Cybern 1988;18(1):183-190.

26. Yager RR. Families of OWA operators. Fuzzy Sets Syst 1993;59:125-143.

27. Calvo T, Mayor G, Mesiar R. Aggregation operators, new trends and applications. Heidelberg, Germany: Physica-Verlag; 2002.

28. Calvo T, Mesiar R, Yager RR. Quantitative weights and aggregation. IEEE Trans Fuzzy Syst 2004;12(1):62-69.

29. Grabisch M, Marichal JL, Mesiar R, Pap E. Aggregation functions. Cambridge, UK: Cambridge University Press; 2009,.

30. Klement EP, Mesiar R, Pap E. Triangular norms. Berlin: Springer-Verlag; 2000.

31. Kolesarova A, Mesiar R, Ruckschlossova T. Power stable aggregation functions. Fuzzy Sets Syst 2014;240:39-50.

32. Mares M, Mesiar R. Information in vague data sources. Kybernetika 2013;49(3):433-445.

33. Wallmann C, Kleiter GD. Degradation in probability logic: when more information leads to less precise conclusions. Kybernetika 2014;50(2):268-283.

34. Merigó JM, Gil-Lafuente AM. The induced generalized OWA operator. Inform Sci 2009;179(6):729-741.

35. Mesiar R, Mesiarová-Zemánková A. The ordered modular averages. IEEE Trans Fuzzy Syst 2011;19(1):42-50.

36. Singh AK, Kishor A, Pal NR. Stancu OWA operator. IEEE Trans Fuzzy Syst 2015;23:13061313. 